\title{
Neutrophils Band Form to Neutrophils Ratio Measurement
}

National Cancer Institute

\section{Source}

National Cancer Institute. Neutrophils Band Form to Neutrophils Ratio Measurement. NCI Thesaurus. Code C120642.

The determination of the ratio of neutrophil band form compared to neutrophils present in a sample. The measurement may be expressed as a ratio or percentage. 